\title{
Superación de agresiones sexuales infanto-juveniles y el sistema judicial: una visión desde los participantes
}

\section{Healing from child and youth sexual assault and the justice system: A view from the participants}

\author{
Ximena Lama, Carolina Gutiérrez, Claudia Capella, Denise Dussert, Loreto Rodríguez, Gretchen Beiza \\ y Daniela Águila
}

Universidad de Chile, Santiago, Chile

\begin{abstract}
Resumen: Este estudio se realiza en el marco de un proyecto de investigación mayor, que estudia cómo se producen los procesos de superación de la experiencia de agresión sexual, tanto para niños(as) y adolescentes, así como para sus adultos responsables y terapeutas. El presente artículo da cuenta de los resultados de los aspectos legales que favorecen y dificultan la superación, incorporando las miradas de niños(as), adolescentes y sus adultos a cargo. La investigación utiliza metodología cualitativa, realizándose entrevistas en profundidad a 27 niños, niñas y adolescentes que culminaron de manera exitosa su proceso terapéutico debido a una situación de agresión sexual y a 18 de sus padres o adultos a cargo. Los principales resultados aluden a que ambos grupos coinciden en visualizar el actuar del sistema judicial como obstaculizador de sus procesos de superación, realizándose distinciones solo en los casos en los cuales los resultados de estas acciones culminan con la condena del agresor, lo que es visualizado positivamente, como protector. Se discuten las implicancias prácticas para el sistema judicial y para las intervenciones psicosociales en estos casos.
\end{abstract}

Palabras clave: abuso sexual, reparación, proceso judicial, juicio, víctimas.
Abstract: This study was developed in the context of a larger research project, which examines the healing process of children and adolescent victims of sexual abuse, as well as their caregivers and psychotherapists. This research shows the results related to legal aspects that favour and hinder the healing process from sexual abuse, considering the opinion of children, adolescents and their caregivers. This study uses a qualitative methodology. Indepth interviews were carried out with 27 children and adolescents who successfully completed the treatment program, 18 of their parents or caregivers were also interviewed. The main results indicate that both groups, children and parents, consider the justice system as the main obstacle in the healing process, except in those cases where the offender is convicted which is positively visualized by the participants as an act of protection. Practical implications for the justice system and psychosocial interventions are discussed.

Keywords: sexual abuse, repair, judicial process, trial, victims.

La presente investigación fue financiada por el programa U-Inicia Concurso de reforzamiento de inserción productiva de nuevos académicos de la Vicerrectoría de Investigación y Desarrollo de la Universidad de Chile, proyecto U-Inicia 5/0612.

Contacto: C. Capella. Departamento de Psicologia. Avenida Capitán Ignacio Carrera Pinto 1045, Ñuñoa, Código Postal 6850331, Santiago, Chile. Correo electrónico: ccapella@u.uchile.cl

Cómo citar: Lama, X., Gutiérrez, C., Capella, C., Dussert, D., Rodríguez, L., Beiza, G. y Águila, D. (2015). Superación de agresiones sexuales infanto-juveniles y el sistema judicial: visión desde los participantes. Revista de Psicología, 24(1), 1-19. http://dx.doi.org/10.5354/0719-0581.2015.36914 


\section{Introducción}

Las Agresiones Sexuales InfantoJuveniles (ASI) son un fenómeno social que ha alcanzado gran relevancia en la actualidad, presentando alta prevalencia a nivel internacional (Pereda, Guilera, Forns y Gómez-Benito, 2009). En Chile se ha descrito que alrededor de un $9 \%$ de niños(as) y adolescentes han sido víctimas de alguna forma de agresión sexual (Unicef, 2012). Sin embargo, es sabido que existe una elevada cifra negra en estos casos, entendida como la proporción de delitos que no son denunciados a la autoridad (Maffioletti y Huerta, 2011), subregistro que alcanzaría cifras cercanas al 10\% (Putnam, 2003).

Por otro lado, han sido descritas ampliamente las consecuencias negativas que genera esta experiencia en las víctimas, presentando diversa sintomatología (Echeburúa y Guerricaechevarría, 2005), e impactando en el desarrollo de los niños, niñas y adolescentes en diferentes esferas (Foster y Hagedorn, 2014). Debido a esta amplia gama de consecuencias, se ha planteado la necesidad de tratamientos psicológicos adecuados para quienes han sido víctimas (Jumper, 1995 citado en Echeburúa y Guerricaechevarría, 2005), principalmente porque las ASI se consideran un factor de riesgo importante para el desarrollo de psicopatologías, tanto en la infancia como en la adultez (Cutajar et al., 2010).

La institución principalmente responsable del tratamiento a víctimas infantojuveniles de ASI en Chile es el Servicio Nacional de Menores (Sename) que provee de una amplia oferta de programas de intervención en estos casos. El objetivo principal de estos programas se relaciona con contribuir a la protección de niños(as) y adolescentes, promoviendo su recuperación integral a través de una terapia de reparación, que les posibilite resignificar los efectos psicosociales que las ASI han generado (Sename, 2012).

Se ha discutido a nivel nacional respecto de la escasa sistematización, claridad, especificidad y profundidad del concepto de terapia de reparación que propone Sename (Martínez, 2012). Capella y Gutiérrez (2014), plantean que este aludiría principalmente a la posibilidad de reparar lo dañado, lo que implica un retorno a un estado de funcionamiento anterior al trauma, poniendo el énfasis de la psicoterapia en superar las consecuencias negativas de la experiencia de agresión sexual $\mathrm{y}$, por tanto, en la remisión sintomática.

Debido a esto, se han buscado nuevas conceptualizaciones que permitan ampliar la mirada acerca del fenómeno (Capella y Gutiérrez, 2014). Desde esta investigación se ha optado por utilizar el concepto de superación, entendiendo que las personas no solo afrontan las consecuencias negativas de la experiencia, sino que son parte de un proceso dinámico, donde es posible que se genere un crecimiento, en la medida en que pueden tener un proceso de cambio positivo y de fortalecimiento al hacer sentido de la experiencia abusiva (Draucker et al., 2011).

Diversos estudios, principalmente con adultos, han investigado cómo las víctimas visualizan este proceso de superación, cuáles son las estrategias que han utilizado para salir adelante y cuáles son los factores que lo favorecen y dificultan (Draucker et al., 2011; Foster y Hagedorn, 2014). De esta forma, han sido mencionados factores personales (tales como estrategias de afrontamiento $\mathrm{y}$ agencia personal), familiares (principalmente el apoyo y credibilidad) y el proce- 
so psicoterapéutico, el cual es identificado como uno de los factores de mayor relevancia en la superación, vinculado a modalidades de tratamiento focalizadas en la experiencia traumática de agresión sexual (Banyard y Williams, 2007; Cohen, Mannarino y Deblinger, 2006; Draucker et al., 2011; Foster y Hagedorn, 2014). Estos factores estarían interrelacionados y se conjugarían dinámicamente frente a la recuperación de la experiencia abusiva.

En esta línea, dentro de los factores que influirían en el proceso de superación, Foster y Hagedorn (2014) dan cuenta de la importancia del proceso judicial en niños, niñas y adolescentes, visualizando que los cambios legales destinados a su protección les habrían provocado desestabilización.

En Chile, paralelo al proceso de psicoterapia, las víctimas usualmente participan de procesos judiciales, tanto a nivel de Tribunales de Familia como de Fiscalía. La denuncia en el proceso penal permitiría tomar posición frente al hecho delictivo (Cortés et al., 2009). De acuerdo con Maffioletti y Huerta (2011), los delitos sexuales representan alrededor de un $1.4 \%$ del total anual de denuncias, dentro del cual un $70 \%$ corresponde a agresiones cometidas contra niños(as) y adolescentes.

En la etapa de investigación, el Ministerio Público de Chile realiza diligencias con el objetivo de obtener pruebas para esclarecer los hechos. En este proceso la víctima es requerida en diversas ocasiones. Una vez que ha finalizado la investigación, el fiscal decide si tiene antecedentes suficientes para acusar al imputado y llegar a la instancia de juicio oral (Cárdenas, Grunert y Vásquez, 2007).

Del total de juicios orales realizados en Chile entre los meses de enero y sep- tiembre de 2014, un 9\% de ellos correspondieron a juicios por causas de delitos sexuales, concluyendo alrededor de un $77 \%$ de ellos en sentencias condenatorias (Ministerio Público de Chile, 2014). No obstante, la mayoría de quienes participan de los procesos no queda conforme con el resultado, lo que es particularmente cierto en aquellos casos en los que la sentencia es absolutoria (Cortés et al., 2009).

A pesar de que niños, niñas y adolescentes son protagonistas de todo este proceso judicial, existen escasos estudios que recojan sus percepciones al respecto (Foster y Hagedorn, 2014).

A nivel internacional, dichos autores refieren que los(as) niños(as) y adolescentes participantes de su investigación habrían experimentado sentimientos de frustración por haber sido entrevistados en numerosas ocasiones, como también aprehensión y ansiedad ante la participación en juicios. Además, plantean vivencias de injusticia, vinculadas principalmente a que las causas no prosperan por falta de evidencia y desconocimiento del paradero del agresor, lo que genera temor a la revictimización.

Más allá de la resolución de los tribunales, Foster y Hagedorn (2014) observan que el paso por el sistema judicial es siempre extremadamente difícil y según se ha podido describir, en ocasiones nocivo. Este fenómeno es conocido como victimización secundaria y alude a las reacciones negativas del entorno frente a la victimización primaria (Boletín $\mathrm{N}^{\circ}$ 9.245-07, 2014), tema que ha generado discusión en Chile.

A nivel nacional, una investigación con niños(as) y adolescentes visualiza que el tránsito por el sistema judicial es dificultoso, en tanto se realizarían una gran 
cantidad de interrogatorios y diligencias, siendo raramente sensibles con quienes deben declarar. Asimismo, los participantes evaluarían el proceso de manera negativa debido a la escasa información con la que cuentan respecto de este (Cortés et al., 2009).

Miranda (2012) plantea que el proceso penal en Chile produciría victimización secundaria a través de las diversas etapas que recorre la víctima, constituyéndose en una vulneración de derecho de acuerdo a lo garantizado por la constitución chilena y los tratados internacionales suscritos (Boletín N 9.245-07, 2014).

En este sentido, se ha planteado como perentorio un proceso que considere la etapa evolutiva de la víctima, velando por el interés superior del(a) niño(a) y/o adolescente. Esto, debido a que la victimización secundaria agravaría el daño psicológico sufrido, obstaculizando el proceso de superación de la experiencia traumática. En este sentido, el proceso judicial sería una experiencia perjudicial para las víctimas, en tanto "pueda convertirse, por sí mismo, en una experiencia lesiva para los derechos de los niños, niñas o adolescentes que se encuentran en un estado de severa fragilidad psicológica” (Boletín N 9.245-07, 2014, p. 3).

A pesar de que se conocen los efectos negativos del paso por el sistema judicial, no se ha estudiado cómo la victimización secundaria afectaría los procesos de superación de las ASI desde la percepción de los participantes, ni tampoco cómo las resoluciones del sistema judicial influyen en la psicoterapia. Este estudio pretende aportar al conocimiento que se tiene al respecto, vinculando las significaciones de niños, niñas, adolescentes y sus adultos responsables, respecto del proceso judicial y la superación de las agresiones sexuales. También releva la visión de los padres, no solo como acompañantes de sus hijos e hijas, sino considerándolos en su rol de víctimas indirectas, visualizando de qué manera el paso por el sistema judicial impacta la propia superación de las agresiones sufridas por sus niños y niñas.

Para ello se utilizó una metodología cualitativa, basada en el análisis de las narrativas de los involucrados, por lo que la apuesta de este trabajo consiste en relevar las voces de los participantes de estos procesos.

\section{Metodología}

Esta investigación es parte de un estudio cualitativo mayor de investigación en superación y psicoterapia con niños(as) y adolescentes que han sido víctimas de agresiones sexuales (Capella, 2012).

La muestra fue de carácter intencionado, seleccionándose casos que cumplían con los criterios de inclusión. Además fue un muestreo teórico, buscándose que los participantes representaran diferentes posiciones a partir de los atributos que caracterizan al colectivo (Valles, 2003).

La muestra corresponde a 27 niños, niñas y adolescentes de entre 8 y 18 años de edad que han sido víctimas de una agresión sexual y que se encontraban en proceso de finalizar $o$ han finalizado un proceso terapéutico vinculado a esta experiencia. Este tratamiento había tenido una duración de entre 10 y 48 meses, con un promedio de 24 meses. En todos los casos el terapeuta reporta que el caso es egresado del centro por el cumplimiento de los objetivos terapéuticos. Las principales características de la muestra se reflejan en la tabla 1 . 
Tabla 1

Tabla de caracterización de la muestra de niños(as) y adolescentes

\begin{tabular}{llc}
\hline Características & \multicolumn{1}{c}{ Categorías } & Frecuencia \\
\hline \multirow{2}{*}{ Edad } & Niños 8-12 años & 12 \\
& Adolescentes 13-18 años & 15 \\
Sexo & Mujeres & 21 \\
& Hombres & 6 \\
\multirow{2}{*}{ Agresor } & Figura paterna & 11 \\
& Otro familiar & 12 \\
& Conocidos & 4 \\
Tipo de abuso & Abuso sexual (acciones sexuales sin & 17 \\
& contacto penetrativo) & 10 \\
Cronicidad del abuso & Violación (contacto sexual penetrativo) & 24 \\
& Repetido o crónico & 3 \\
\hline
\end{tabular}

Para conformar la muestra de adultos responsables, se seleccionó a los padres o principales cuidadores de los(as) niño(as) o adolescentes participantes del estudio, que los habían acompañado durante todo el tratamiento, participando activamente de este. Es este contexto fue posible realizar entrevistas a 18 padres o adultos responsables, del total de casos (ver la tabla 2).

La muestra se obtuvo desde tres programas de atención especializada en maltrato y agresión sexual de la Región Metropolitana: uno es CAVAS (Centro de Asistencia a Víctimas de Atentados Sexuales) en su área de Reparación, dependiente de la Policía de Investigaciones de Chile; y los otros dos proyectos pertenecen a la Fundación Ciudad del Niño. El criterio de número de participantes fue determinado a través del Método de Saturación de Información (Valles, 2003), que implica que la recolección de datos se detiene cuando no emerge nueva información que resulte significativa. Para la recolección de datos, se realizaron entrevistas en profundidad para rescatar la subjetividad de los participantes (Valles, 2003). En cuanto a las áreas temáticas, se elaboraron guiones de entrevista diferenciados para niños, niñas, adolescentes y adultos a cargo, en los cuales se les invitó a realizar una narrativa acerca de cómo visualizaban el proceso de psicoterapia y superación y cuáles eran los factores que lo facilitaban y dificultaban. Dentro de esto, se incorporaron preguntas específicas de la percepción del proceso judicial y de los resultados de este, además de la visualización de su impacto en la superación. Los guiones de los adultos a cargo, por su parte, incorporaban preguntas tanto respecto del proceso de superación y psicoterapia llevado a cabo por sus hijos(as), así como de sus propios procesos de superación de la experiencia de sus niños. Los guiones de entrevista estaban conformados por preguntas abiertas, las que permitieron la profundización de las diversas temáticas mencionadas $\mathrm{y}$, complementariamente se realizaban preguntas aclaratorias en función de los tópicos que los propios entrevistados $\mathrm{y}$ entrevistadas iban señalando. 
Tabla 2

Tabla de frecuencia de adultos responsables

\begin{tabular}{llcc}
\hline Características & \multicolumn{1}{c}{ Categorías } & Número & Entrevistados \\
\hline & $\begin{array}{l}\text { Padre, madre o ambos } \\
\text { padres }\end{array}$ & 15 & 12 \\
$\begin{array}{l}\text { Figura cuidadora del(a) } \\
\text { niño(a) o adolescente }\end{array}$ & $\begin{array}{l}\text { Otra figura cuidadora } \\
\text { (abuela, tía, madre } \\
\text { adoptiva, etc.) }\end{array}$ & 7 & 6 \\
& Hogar de protección & 5 & 0 \\
\hline
\end{tabular}

Las entrevistas, con una duración aproximada de una hora por cada participante, fueron realizadas por las integrantes del equipo de investigación, de manera paralela y por separado a niños(as) o adolescentes y sus adultos a cargo. Estas se realizaron en los centros en los que los participantes habían cursado sus terapias, en un periodo de un mes antes, o bien, después de finalizado el tratamiento. Previamente a la realización de las entrevistas, los adultos firmaron un consentimiento informado, mientras que niños, niñas y adolescentes fueron informados acerca de la investigación y otorgaron su asentimiento. El proyecto de investigación fue aprobado por el Comité de Ética de la Investigación en Ciencias Sociales y Humanidades de la Universidad de Chile. Durante todo el proceso se resguardó el bienestar de los participantes, así como la confidencialidad de la información.

Para el análisis de los datos, todas las entrevistas fueron grabadas y posteriormente transcritas. El análisis se realizó mediante la técnica de análisis narrativo, ya que se ha planteado su utilidad para comprender la perspectiva de los participantes, en tanto estudia los relatos que las personas construyen de sus experiencias, dándoles significados a estas, particularmente las historias personales que incorporan dimensiones de temporalidad y subjetividad (Capella, 2013; Riessman, 2008).
Se realizó un análisis temático narrativo (Riessman, 2008) de cada una de las entrevistas, destacando los significados en torno a los temas propuestos, así como también de las temáticas emergentes. Luego, se procedió a realizar un análisis intercasos, en el cual se identificaron similitudes y diferencias, las cuales fueron integradas y organizadas en unidades temáticas de diferentes niveles de integración. Las unidades temáticas surgieron del análisis, no encontrándose definidas previamente.

En primer lugar, el análisis intercasos se concentró en revisar por separado todos los casos de niños(as), adolescentes y adultos. Luego, se apreciaron las convergencias y divergencias entre estos tres grupos. Finalmente, se organizaron y ordenaron las diferentes unidades temáticas en distintos ejes, para dar coherencia a los resultados y respuesta al objetivo de investigación.

Cabe señalar que todo el proceso de análisis fue realizado en equipo, contrastando y triangulando los resultados con los de los otros investigadores. A su vez, los resultados de la investigación fueron presentados a expertos en la temática del tratamiento de las agresiones sexuales, validándolos (Riessman, 2008) sobre la base de sus conocimientos y experiencia en relación con el tratamiento y proceso 
judicial en niños y adolescentes víctimas. De esta forma, se llevó a cabo la triangulación de la información por medio de la contraposición y comparación constante de diferentes miradas hacia el objeto de estudio, con lo cual se favorece el rigor y la calidad de los resultados (Cornejo y Salas, 2011).

\section{Resultados}

Se exponen las significaciones del impacto del proceso judicial en los procesos de superación de niños, niñas y adolescentes que han sido víctimas de agresiones sexuales y sus padres o adultos a cargo. Estas visiones son convergentes en muchos de los aspectos señalados, por lo que se presentan en forma conjunta, realizándose -cuando sea pertinente- las distinciones necesarias. Se utilizan citas de las entrevistas para ejemplificar los análisis realizados, usándose pseudónimos para resguardar la confidencialidad.

Cabe señalar que se observan conceptualizaciones más abstractas y específicas en adolescentes y adultos, y en $\operatorname{los}($ as) niños(as) percepciones más generales, lo que se comprende a partir de sus aspectos evolutivos. Además, la mayoría de los participantes se refiere al sistema judicial y a los procesos penales como a un todo, sin hacer distinciones entre procesos en los Tribunales de Familia y en la Fiscalía.

Es importante mencionar que algunos entrevistados no hacen muchas referencias al proceso judicial, especialmente niños, niñas y adolescentes, al mismo tiempo que muestran malestar al referirse a la temática, o bien, mantienen distancia del proceso. Esto les permite protegerse de las emociones que les genera la situación, señalando que son los padres o adultos significativos quienes se hacen cargo de las diligencias.
Mi mamá tiene todos los papeles en la casa, (...) al final no hicieron nada. Así que, por eso no me gusta hablar de eso. (Vaitea, 17 años).

A partir de las narrativas de niños(as), adolescentes y sus padres o adultos significativos, es posible distinguir tres ejes temáticos principales en la valoración realizada de la intervención del sistema judicial, los que se describen en la tabla 3. Por una parte se destaca el proceso judicial del cual son parte, por otra los resultados de este proceso $\mathrm{y}$, finalmente, el impacto de ambos en la superación de las agresiones sexuales.

\section{Significaciones del proceso judicial}

Desde la mayoría de las narrativas aparece una visión predominantemente negativa del proceso judicial por el que deben transitar tras la develación de la situación de agresión sexual. Esta visión negativa se vincula tanto a las prácticas del sistema judicial, como al desarrollo del proceso y a las acciones de los operadores de justicia.

En términos de las prácticas, se valora negativamente la gran cantidad de interrogatorios, la importancia de la protección al momento de la declaración y la escasa información que se recibe desde el sistema. Así, las prácticas durante el proceso judicial son evaluadas como vulneradoras por los constantes interrogatorios a los que son sometidos niños, niñas y adolescentes los cuales, de acuerdo a los entrevistados, no aportarían mayores antecedentes a la investigación, sino más bien serían una repetición de la misma información.

Porque vez que me citan es pa'... pa' declarar lo mismo una y otra vez y ya he tenido que ir más de cuatro veces a contar la misma, la misma tontera. (Karen, 18 años). 
Tabla 3

Tabla de resultados en ejes y unidades temáticas

\begin{tabular}{|c|c|c|}
\hline Ejes temáticos & Unidades temáticas & Descripción de subunidades \\
\hline \multirow{7}{*}{$\begin{array}{l}\text { Significaciones del proceso } \\
\text { judicial }\end{array}$} & \multirow{3}{*}{$\begin{array}{l}\text { Prácticas del sistema } \\
\text { judicial }\end{array}$} & Constantes interrogatorios \\
\hline & & $\begin{array}{l}\text { Necesidad de protección al } \\
\text { declarar }\end{array}$ \\
\hline & & Desinformación \\
\hline & \multirow[t]{2}{*}{ Desarrollo del proceso } & Lento, difícil y cansador \\
\hline & & Ineficiente y decepcionante \\
\hline & \multirow{2}{*}{$\begin{array}{l}\text { Acciones de los } \\
\text { operadores }\end{array}$} & Atención poco adecuada \\
\hline & & Trato comprensivo \\
\hline \multirow{6}{*}{$\begin{array}{l}\text { Significaciones en relación a } \\
\text { los resultados del proceso } \\
\text { judicial }\end{array}$} & \multirow[t]{3}{*}{ Expectativas } & Castigo \\
\hline & & Protección \\
\hline & & Escucha y/o credibilidad \\
\hline & \multirow{2}{*}{$\begin{array}{l}\text { Valoración negativa } \\
\text { de los resultados }\end{array}$} & No se cumplen expectativas \\
\hline & & Sensación de injusticia \\
\hline & $\begin{array}{l}\text { Valoración positiva de } \\
\text { los resultados }\end{array}$ & Se cumplen las expectativas \\
\hline \multirow{3}{*}{$\begin{array}{l}\text { Impacto del sistema judicial } \\
\text { en la superación de las agre- } \\
\text { siones sexuales }\end{array}$} & \multirow[t]{2}{*}{$\begin{array}{l}\text { Obstaculizador para el } \\
\text { proceso de superación }\end{array}$} & $\begin{array}{l}\text { Procesos y resultados judiciales } \\
\text { obstaculizan }\end{array}$ \\
\hline & & Sistema obstaculiza psicoterapia \\
\hline & $\begin{array}{l}\text { Favorecedor para el } \\
\text { proceso de superación }\end{array}$ & $\begin{array}{l}\text { Derivación a psicoterapia } \\
\text { Condena del agresor }\end{array}$ \\
\hline
\end{tabular}

A partir de esto, aparece la idea de contar con espacios que puedan proteger a las víctimas al momento de declarar.

Entrevistadora: ¿Qué podrían hacer ellos (jueces)?

Niña: Que, eh, si se siente incómodo por ejemplo, que ellos estén aparte. Como lo hicieron conmigo, ellos estaban en otro lugar (en el juicio oral). (Alejandra, 8 años).

Cabe destacar que para muchos(as) niños(as) y adolescentes la idea de declarar produce miedo, por el temor de ver al agresor y reiterar el contacto con esta figu- ra, presentando gran incertidumbre respecto de los procedimientos judiciales.

Claro, (...) que él podía, no sé, pararse, ir al lado mío, ese era el miedo que tenía. (Jennifer, 12 años).

No me gustaría ir, porque no quiero verlo a él (agresor). (Katherine, 11 años).

Esta incertidumbre y poca claridad respecto del proceso que en el caso de niños y niñas podría entenderse a partir de sus recursos evolutivos, también se aprecia en los adolescentes y adultos, que mencionan 
que habría una constante desinformación por parte del sistema judicial respecto de los procedimientos llevados a cabo. Esto los hace sentir pasados a llevar y no considerados, generándoles angustia:

No sé, que me explicaran a dónde me llevaban, porque yo no cachaba. (Jorge, 15 años).

Que son muy largos, muy angustiantes, entonces tú estás en estrés continuo, porque tú no sabes. (Eulalia, abuela de Alejandra, 8 años).

En vinculación con lo anterior y respecto del desarrollo del proceso judicial, uno de los aspectos evaluados más negativamente se refiere a una visión del proceso como difícil, lento y cansador.

Que hagan las cosas más rápidas, son muy lentos, demasiado (...) porque no puede ser que después de que uno ponga la denuncia o sea... pase tanto tiempo después. (Jimena, 17 años).

Junto con esto, el desarrollo del proceso es valorado negativamente por la mayoría de los adultos, siendo apreciado como ineficiente y decepcionante.

Yo esperaba que la justicia hiciera algo (...) pensé que iba a ser más rápido, o sea, nosotros teníamos que andar llamando y yendo muchas veces, y nos decían que no, no está (...); muchas veces nos citaban y nada. (Edith, madre de Vaitea, 17 años).

El proceso judicial ha sido pésimo, yo creo que si tuviera que ponerle nota le pongo un cero. (Laura, madre de Agustina, 13 años).

Dentro del proceso judicial también se valoran negativamente las acciones de los operadores de justicia. En su mayoría, los entrevistados coinciden en dar cuenta de una atención poco adecuada por parte de dichos operadores (carabineros, pericias, Fiscalía, Servicio Médico Legal, etc.), no haciendo una distinción espontánea respecto de las diferentes instancias. La atención se percibe maltratadora por el actuar de los propios intervinientes, quienes son evaluados como desinteresados, poco considerados e incluso en algunos casos, violentos. También los lugares de atención son valorados como poco adecuados. Esto se observa más directamente en lo señalado por adolescentes, donde aparece la incomodidad frente a este actuar y la necesidad de mayor especialización en los operadores.

(Respecto de qué debiera cambiar) Que no te hiciera tanto daño el proceso... la señora que te toma la declaración no es muy simpática, no es un lugar apto (...) deberían adecuarse un poco, un chiquitito, lo que puedan así, por lo menos poner como personas más capacitadas para eso. (Dominga, 17 años).

Sé que ellos son duros, pero no todos somos iguales y eso que no les gustaría que sus hijos estuviesen en ese momento, que le estén tratando mal o que te digan que te van a pegar una cachetada si no querí hablar. (Jorge, 15 años).

Aun cuando lo anterior se refleja de manera mayoritaria, algunos participantes perciben un trato comprensivo por parte de algunos operarios del sistema.

Entrevistadora: El juez, ¿cómo te trató? Niño: Bien (...) porque me decía muchas cosas. (Daniel, 9 años).

Por medio de esta visión general negativa del proceso judicial por parte de niños(as) $\mathrm{y}$ adolescentes se desprende un malestar e 
incomodidad vinculado a su participación en este e incluso una percepción de haber sido vulnerado por el sistema.

El sistema judicial ha vulnerado todos mis derechos, porque yo me siento vulnerada, yo me siento horrible ahora. (Dominga, 17 años).

Significaciones en relación con los resultados del proceso judicial

Si bien hay una evaluación negativa compartida entre los entrevistados respecto del proceso judicial, la valoración en cuanto a los resultados da cuenta de percepciones, ya sean negativas o positivas, las cuales se relacionan con la satisfacción de sus expectativas acerca del sistema judicial.

Respecto de las expectativas, destaca en todos los entrevistados la necesidad de castigo para el agresor por los actos cometidos, esperando que exista un esfuerzo del sistema judicial a fin de que dicho objetivo se cumpla.

Que a los que hicieron eso, los castigaran (...) encerrándolos en la cárcel. (Soledad, 9 años).

Dicho castigo es asociado por los(as) niños(as) a la protección, vale decir, no tener nuevos contactos con el agresor, lo que podría estar vinculado a las características evolutivas de este grupo etario, en donde la cárcel es la forma concreta y comprensible de hacerlo efectivo.

Por otro lado, la posibilidad de que no se cumpla esta expectativa de protección genera miedo, especialmente en los niños y niñas, en tanto temen que a partir de las diligencias solicitadas se restablezca algún contacto con el agresor y/o no se desplieguen las acciones de protección esperadas por ellos y que permitan la interrupción de la agresión, especialmente cuando la figura del agresor es un familiar.

Para decirles que me quiero quedar con mi mamá y no con mi abuelo (agresor) (...) me sentía mal (...) tenía miedo porque pensaba que el juez me iba a mandar donde mi abuelo. (Isidora, 8 años).

Adolescentes y adultos también visualizan esta necesidad de castigo y protección, asociada a la no ocurrencia de una revictimización, ni agresiones a otros, como también vinculada con la necesidad de protección emocional.

Metiéndolos presos... para que no, no haya más casos así como de abuso. (Teresa, 12 años).

Que la persona no se acerque, porque hay personas que siguen viendo a los niños, a lo mejor a ellos no les gusta (...) y que ellos estén pendientes de que si realmente es dañino ver a esa persona. (Marta, madre de Mariana, 15 años).

Esta expectativa de protección resulta tan relevante, que en algunos casos surge la apreciación de que la superación no está completa en la medida en que no se ha obtenido la protección esperada desde el sistema judicial.

Superado entre comillas porque, siempre con el temor de que él aparezca. (Ana, madre de Cecilia, 13 años).

Incluso ahora como que a veces pienso y digo: "me puedo topar con él en cualquier parte", no sé, es como... no me siento aliviada. (Ágata, 15 años).

Junto con esto, como expectativa, para los entrevistados es importante que se les 
otorgue escucha y/o credibilidad, pues esta podría favorecer el proceso de superación mediante la condena y/o medidas de protección, aun cuando también puede generar temor.

Entrevistadora: ¿y te gustaría ir a contar lo que pasó?

Niña: Sí (...), si entienden más el caso (...) lo pueden resolver. (Katherine, 11 años).

Claro y de que le vayan a creer a él, eso era el miedo que yo tenía. (Jennifer, 12 años).

No obstante, cuando esta expectativa de escucha y/o credibilidad, no se cumple, se releva la percepción de sentirse poco escuchados y considerados por un sistema judicial que les genera malestar y no facilita sus procesos de superación.

Que venga una persona que nunca ha hablado con la Isidora, nunca ha estado con ella, que diga no que a lo mejor la niña se lo imaginó, yo creo que no corresponde. (Ivette, abuela de Isidora, 8 años).

La mayor parte de las narrativas dan cuenta de una valoración negativa de los resultados del actuar del sistema judicial, pues perciben que pese a todos los esfuerzos que realizan, los resultados no son los esperados, no se cumplen sus expectativas, dando cuenta de que "no se hizo nada".

No sé, yo siento que con lo que me pasó no se hizo justicia, siendo que igual fue injusto, siendo que me habían hecho pruebas, me hicieron ir a tanta parte y después para que al final dijeran que no habían, ¿cómo se llama?, pruebas o no justificaba lo que había pasado. (Ágata, 15 años).
A él nunca lo buscaron, nunca lo notificaron, nunca nada, nosotros somos amigos de la mamá de él, y sabíamos que no, nunca le llegó notificación, nunca lo fueron a ver. (Marcos y Jazmín, padres de Soledad, 9 años).

Todo lo anterior les genera a los adultos una fuerte sensación de injusticia, vinculada directamente al mal actuar respecto de sus casos, pero que se extiende, decepcionándolos en general respecto del sistema judicial.

Las leyes son malas aquí en Chile, (...) el sistema es malo. (Jaime, padre de José 8 , años).

El proceso judicial lo vivimos así como que, decepcionada de la justicia. (Ivette, abuela de Isidora, 8 años).

Es posible visualizar en varios adultos la necesidad de que si la justicia no viene desde el sistema judicial, provenga de alguna otra instancia, ya sea desde lo más espiritual o hasta ejercerla con "las propias manos".

Sí, la justicia divina llegó ya, la justicia del hombre no existe, pero la justicia divina sí (...) cuando le sucedió esto a mi hijo, no sabía que... no sabía qué hacer (...) matarlos a los que le hicieron daño a mi hijo, pero la fiscal me dijo que qué sacaba. (Jaime, padre de José, 8 años).

Cabe destacar que en solo cuatro casos de la muestra se establece una condena de cárcel a los agresores. En estos casos existe una visualización positiva del resultado del actuar judicial, en tanto cumple las expectativas de protección y castigo.

Niño: Yo lo encuentro como un alivio de que pague por lo que hizo. 
Entrevistadora: ¿Un alivio de...? ¿qué te alivia?

Niño: Del miedo de que podría estar caminando por mi calle. (Agustín, 12 años).

Ayuda, porque ella sabe que está preso, saber que está detenido, que fue castigado por lo que le hizo. (Eulalia, abuela de Alejandra, 8 años).

Impacto del sistema judicial en la superación de las agresiones sexuales

Se aprecia que existe una tendencia en los entrevistados a dar cuenta del sistema judicial -tanto de sus procesos, como de sus resultados-, como un obstaculizador importante para el proceso de superación de las agresiones sexuales.

(...) y ellos mismos se encargan de que te cueste superar eso, (...) los mismos tribunales, todo. (Karen, 18 años).

Entonces es traumante, ese es otro proceso que uno aparte del tema de lo que le pasó a las niñas, uno tiene que superar que la justicia es nula, entonces eso también necesita un periodo como psicológico como de entender y adaptarse. (Laura, madre de Agustina, 13 años).

Adolescentes y adultos, principalmente, destacan la psicoterapia como favorecedora del proceso de superación, pero que el sistema judicial la obstaculizaría, enlenteciendo el proceso de cambio dentro de esta y, por ende, la superación de las agresiones sexuales, haciendo incluso que esta retroceda.

Tuvimos que ir a Fiscalía y ella tenía que tratar de recordar con el fiscal, entonces fue truncando ahí a veces el proceso que llevaba acá (psicoterapia), que había adelantos... retroceder y ella salía angustiada de ahí (Eulalia, abuela de Alejandra, de 8 años).

Lo anterior es visualizado por algunos participantes como paradójico, en tanto es el mismo sistema judicial el que usualmente los deriva a psicoterapia a fin de superar la experiencia traumática.

Claro po', qué sacan con mandarnos a proceso psicológico, si nos van a estar llamando a cada rato pa' contar la misma historia y llamando a tribunales a testificar siempre, entonces no tiene sentido, se contradicen solos. (Karen, 18 años).

En ese sentido, aun cuando los adolescentes y sus adultos responsables indican el efecto iatrogénico del sistema judicial, valoran la derivación que dicha instancia realiza a psicoterapia. Todos los integrantes de la muestra consideran que este espacio es positivo en tanto favorecedor de la superación de las agresiones sexuales.

(Aludiendo a la importancia de la derivación y el apoyo recibido en el espacio terapéutico) Como le digo, la tiraron mucho (Fiscalía), por muchos lados (pericias) y la Fiscalía tendría que haberla tirado primero hacia acá (terapia) y después a los otros lados donde la mandaban. (Edith, madre de Vaitea, de 17 años).

A pesar de que la mayor parte de los entrevistados evalúa negativamente el resultado judicial, en algunos casos en donde este culmina con la condena del agresor, se visualiza que esta favorece la superación.

(Aludiendo a que le ayuda a la sanción) De que ahora... él, él si ya está en la cárcel sería genial pa' mí (voz de risa). $\mathrm{Y}$, todas esas cosas, me puse contento 
cuando dijeron que se iba ir a la cárcel. (Agustín, 12 años).

Entrevistadora: Y la Alejandra ¿cómo se sintió con esta resolución de juicio? Eulalia: Feliz, muy contenta. Claro, de ahí como que ella ha despertado (...) o sea, como queriendo decir "ehhh, este tipo está adentro, no me va a molestar, no me va a pasar nada", como protegida también.

Entrevistadora: $¿ \mathrm{Y}$ a ustedes como familia?

Eulalia: También po', obvio, aunque encontramos que la pena no fue muy... pero se hizo justicia como sea. (Eulalia, abuela de Alejandra, de 8 años).

\section{Conclusiones}

A partir de los resultados obtenidos, es posible dar cuenta de una evaluación mayoritariamente desfavorable del sistema judicial en los participantes, tanto en términos de sus procesos y resultados, como de las implicancias que tiene para la superación de las agresiones sexuales. A partir del análisis de las narrativas de los entrevistados podemos observar que lo que se evalúa negativamente son prácticas ineficaces, un trato inadecuado por parte de los operadores, un desarrollo lento del proceso y la inexistencia de condena para el agresor: estos elementos se conjugan en la percepción de los entrevistados, quienes evalúan por lo general de manera negativa el sistema judicial. Pese a ello, en los casos en los que existe una condena al agresor y un adecuado trato por parte de los operadores, se valora positivamente el rol del sistema judicial en la superación. Entonces, podemos concluir que lo que influye negativamente en el proceso de superación son las prácticas inadecuadas e ineficientes como parte del paso por el sistema judicial del cual son parte las víctimas y sus familias.

Tal como se muestra en la figura 1, que organiza las principales conclusiones, el sistema judicial influenciaría negativamente el proceso de superación de las agresiones sexuales desde la visión de los(as) niños(as), adolescentes y sus adultos a cargo. Sin embargo, existen excepciones que indican cómo ciertas condiciones lo favorecen.

\section{PROCESO DE SUPERACIÓN}

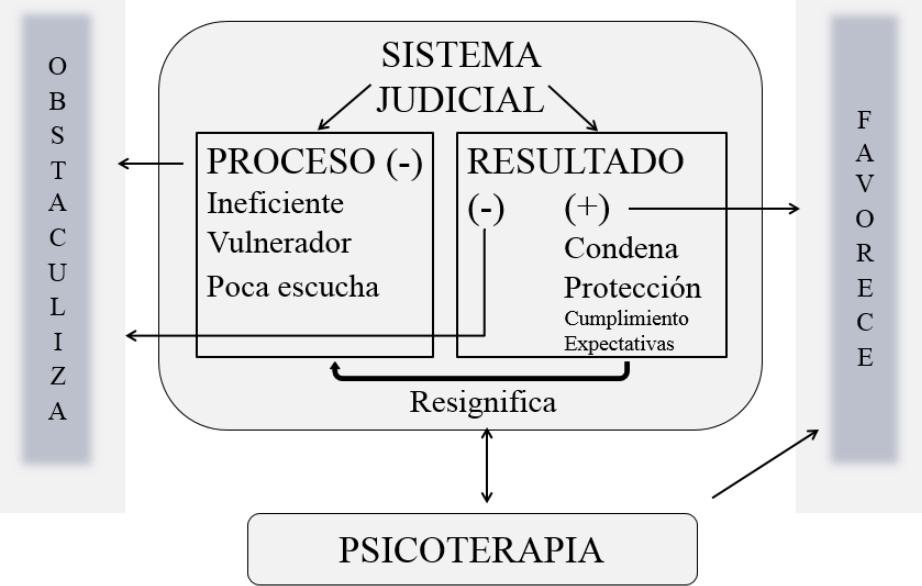

Figura 1. Proceso de superación y sistema judicial. 
El proceso judicial que se vincula con todas las acciones previas a establecer una sentencia es considerado por los entrevistados como difícil y cansador, puesto que les demanda una serie de acciones que evalúan como lentas e ineficientes. Esta percepción, sumada a la desinformación y a las prácticas inapropiadas por parte de los operadores del sistema de justicia, les genera incertidumbre acerca de lo que sucederá a partir de dicho actuar. Los entrevistados se sienten escasamente escuchados y creídos, e incluso desprotegidos durante el proceso judicial.

A este respecto, un estudio en el contexto chileno realizado por Guerra, Viveros, Calvo, Canessa y Mascayano (2011) plantea una visualización del sistema judicial en donde "la víctima es considerada un 'objeto de derecho' que debe cooperar con la justicia para el establecimiento del delito" (p. 20). Se aprecia en el presente estudio que los entrevistados dan cuenta de esta mirada del proceso judicial hacia ellos, durante el cual se sienten poco protegidos.

Respecto del resultado judicial, se aprecia por una parte, una valoración predominantemente negativa, asociada con la percepción de ineficiencias del sistema y los escasos resultados que este otorga en relación con las expectativas de credibilidad, protección y castigo. Esto genera sentimientos de decepción, escasa retribución ante los esfuerzos realizados durante todo el proceso previo y una intensa percepción de injusticia.

Tal como plantea el estudio de Cortés et al. (2009) - cuyos resultados coinciden en términos generales con los encontrados en esta investigación-, muchas veces las motivaciones para denunciar se relacionan con la necesidad de que se haga justicia, apreciando que en tanto esta expectativa no sea cumplida por el sistema judicial, habría una interferencia negativa que dificultaría dar sentido a la experiencia de agresión sexual.

Por otra parte, un grupo menor de participantes destaca aspectos positivos del sistema judicial, relacionados directamente con condenas de cárcel al agresor sexual (cuatro casos). A pesar de que la evaluación del proceso judicial en estos casos también es principalmente negativa, los entrevistados logran resignificarlo, matizando las dificultades y los esfuerzos implicados, dado lo favorable del resultado obtenido. Estos participantes valoran este resultado como favorecedor del proceso de superación, ya que cumple con las expectativas de validar los dichos de niños y niñas que han sido agredidos sexualmente, se les protege y, al mismo tiempo, castiga a los agresores.

Cabe destacar que la visión negativa del proceso judicial, desde los entrevistados, afecta no tan solo la superación, sino también la psicoterapia, en tanto la enlentece o genera retrocesos para dar sentido a la experiencia abusiva. La superación es un proceso dinámico durante el cual se genera un crecimiento a partir de darle sentido a la experiencia de agresión sexual (Draucker et al., 2011), y es apoyado por la psicoterapia (Foster y Hagedorn, 2014), así como por otros factores que, como se han visto en las investigaciones, favorecen y dificultan la superación (Banyard y Williams, 2007; Cohen et al., 2006; Draucker et al., 2011). El proceso judicial, de acuerdo con los participantes, se convierte en un obstaculizador para darle sentido a la experiencia. Esto confirma la importancia del proceso judicial -junto con otros factores- para la superación de la experiencia de agresión sexual, tal como plantea el estudio de Foster y Hagedorn (2014). 
El presente artículo da luces respecto de las necesidades y expectativas de la población infanto-juvenil que ha sido víctima y de sus adultos a cargo, siendo perentorio poder realizar modificaciones, escuchando las voces de los involucrados, en un intento de favorecer sus procesos de superación de las agresiones sexuales sufridas.

En este sentido, una implicancia se vincula con la relevancia de entregar, desde los operadores del sistema judicial, mayor información a los(las) niños(as), adolescentes y a sus familias respecto de los procedimientos que se llevarán a cabo durante el proceso judicial, sus tiempos, y posibles resultados. Esto, con el fin de poder ajustar las expectativas y así tener mayor claridad acerca de la instancia de la cual están siendo parte.

En relación con lo anterior, algunos autores a nivel internacional (Estrada, 2012; Michels, 2009) han relevado la importancia del acompañamiento y la preparación para las diligencias judiciales con los niños y niñas que han sido agredidos sexualmente, por parte de equipos psicosociales debidamente capacitados, para proteger los derechos de las víctimas. Esto favorecería el tránsito por el sistema, el testimonio y los protegería de potenciales daños causados por la participación en el proceso judicial. Se alude a un acompañamiento tanto en las primeras declaraciones, como en el afrontamiento de situaciones estresantes como juicios orales, lo que en un estudio escocés (Plotnikoff y Woolfson, 2001) es valorado positivamente por los propios niños, quienes señalan estar mejor informados acerca de qué y cómo responder en la corte y de lo que puede ocurrir.

Pensando en las implicancias para las intervenciones psicosociales, se requiere que los profesionales que trabajan en el ámbito de la psicoterapia, en los distintos momentos del proceso de intervención, aborden la interferencia del sistema judicial en términos de las emociones generadas en los(las) niño(as), adolescentes y sus adultos a cargo, con las diligencias, las solicitudes o el actuar del sistema, así como también apoyar en la entrega o entendimiento de información respecto del proceso judicial. Esto implica esfuerzos adicionales para ayudar en este tránsito por el sistema judicial, intentando realizar un acompañamiento que permita atenuar sus efectos y favorecer la resignificación del paso por este sistema y, por ende, la superación de la agresión sexual.

Por otra parte, el presente estudio y la visualización predominantemente negativa de los entrevistados, insta a los operadores del sistema judicial a realizar acciones con el fin de mejorar el trato hacia las víctimas infanto-juveniles y sus familias, otorgando mayor y mejor calidad de información, a realizar cambios en las diligencias, a fin de que el recorrido por el sistema judicial favorezca la superación de las agresiones sexuales. En este sentido, tal como propone el Boletín $\mathrm{N}^{\circ}$ 9.245-07 (2014), se hace necesario reformar el sistema judicial, adecuándose a los requerimientos de esta población. Sin embargo, en Chile no existen protocolos de entrevistas basados en evidencia empírica, ni capacitaciones sistemáticas para los entrevistadores en el desarrollo de competencias específicas para que puedan llevar a cabo entrevistas efectivas y no revictimizantes.

Estudios internacionales han destacado la importancia de los protocolos de entrevista, así como la capacitación a los profesionales que están en contacto con niños, niñas y adolescentes dentro del proceso judicial, planteándose la necesidad de implementar entrevistas videograbadas por 
parte de personal debidamente capacitado, dado que esto disminuye al mínimo la victimización secundaria, sin descuidar los objetivos de la investigación penal (Lamb, Orbach, Hershkowitz, Esplin y Horowitz, 2007; Powell, 2008). En esta línea, a nivel nacional, el Núcleo de Investigación Infancia y Justicia (2015) de la Universidad de Chile se encuentra desarrollando una investigación aplicada que pretende diseñar y validar un protocolo de entrevista y un programa de capacitación y entrenamiento de entrevistadores.

Junto con esto, se debiera considerar la existencia de un equipo multidisciplinario en los contextos judiciales, con profesionales del ámbito legal y social (Lipovsky y Stern,. 1997). Esto, con el fin de ir estableciendo un diálogo entre el mundo psicológico, social y el judicial, creando líneas de actuación conjunta que permitan contribuir a una protección efectiva de las víctimas de abuso sexual en la infancia (Echeburúa y Subijana, 2008).

Aun cuando este estudio tuvo diversas limitaciones, tales como el restringido número de la muestra y la escasa variabilidad de centros participantes, se presenta como un aporte a la literatura al dar cuenta del impacto negativo del sistema judicial para el proceso de superación de agresiones sexuales en niños, niñas y ado- lescentes. En este sentido, otra de las limitaciones de esta investigación es que hubo una predominancia de casos con resultados judiciales valorados negativamente por parte de los entrevistados. Es posible que de haber tenido una muestra con mayor cantidad de casos con resultados de condena al agresor, la visión primordialmente negativa del sistema judicial se hubiera matizado. Esto, en la medida en que los resultados de la investigación enfatizan cómo el tránsito por el proceso judicial impacta negativamente en los procesos psicoterapéuticos y de superación, pero que puede ser también un favorecedor en tanto los resultados judiciales se ajusten a las expectativas de castigo y protección de niños(as), adolescentes y sus familias.

Por otro lado, el estudio también aporta en la medida que considera a los padres o adultos a cargo como participantes activos en el proceso judicial, enfatizando el impacto que este genera tanto en sus hijos e hijas como en ellos como víctimas indirectas. De esta forma, permite ir visualizando la necesidad de incluir las voces de niños, niñas, adolescentes y sus figuras a cargo en la construcción de políticas públicas, como sujetos protagonistas de sus procesos, contribuyendo a incentivar y favorecer el bienestar de la población infanto-juvenil.

\section{Referencias}

Banyard, V. \& Williams, L. (2007). Women's voices on recovery: A multi-method study of the complexity of recovery from child sexual abuse. Child Abuse \& Neglect, 31, 275-290. http://dx.doi.org/10.1016/j.chiabu.2006.02.016

Boletín $\mathrm{N}^{\circ}$ 9.245-07. Regula entrevistas grabadas en video y otras medidas de resguardo a menores de edad víctimas de delitos sexuales. Primer trámite constitucional. Senado, Santiago, Chile. Recuperado de http://www.camara.cl/pley/pdfpley.aspx?prmID=9455\&prmTIPO=INICIATIVA 
Capella, C. y Gutiérrez, C. (2014). Psicoterapia con niños/as y adolescentes que han sido víctimas de agresiones sexuales: sobre la reparación, la resignificación y la superación. Psicoperspectivas, 13(2), 93-105. http://dx.doi.org/10.5027/PSICOPERSPECTIVAS-VOL13-ISSUE3- FULLTEXT-348

Capella, C. (2012). Proceso de superación de las agresiones sexuales: narrativas de los niños/as y adolescentes que han sido víctimas, sus padres y sus psicoterapeutas (Proyecto de Investigación U-Inicia 5/0612). Santiago de Chile: Vicerrectoría de Investigación y Desarrollo, Universidad de Chile.

Capella, C. (2013). Una propuesta para el estudio de la identidad con aportes del análisis narrativo. Psicoperspectivas, 12(2), 117-128.

http://dx.doi.org/10.5027/PSICOPERSPECTIVASVOL13-ISSUE2-FULLTEXT-281

Cárdenas, C., Grunert, S. y Vásquez, M. (2007). La víctima en el proceso penal: una propuesta de abordaje para la fase de resolución judicial. En Centro de Atención de Víctimas de Delitos Violentos (Ed.), Atención a víctimas de delitos violentos, reflexiones desde la práctica (2001-2007) (pp. 71-92). Viña del Mar: RIL Editores.

Cohen, J. A., Mannarino A. P., \& Deblinger, E. (2006). Treating trauma and traumatic grief in children and adolescents. New York: Guilford Press.

Cornejo, M. y Salas, N. (2011). Rigor y calidad metodológicos: un reto a la investigación social cualitativa. Psicoperspectivas, 10(2), 12-34. http://dx.doi.org/10.5027/PSICOPERSPECTIVAS-VOL10-ISSUE2-FULLTEXT-144

Cortés, F., Chía, E., Fernández, H., Inzunza, C., Manzi, F., Peña, F. y Hein, A. (2009). Percepción de los procesos de investigación y judicialización en los casos de agresiones sexuales infantiles en las regiones Metropolitana, de Valparaíso y del Bío-Bío (Informe de estudio para la Fundación Amparo y Justicia). Santiago de Chile: Mide UC, Pontificia Universidad Católica de Chile.

Cutajar, M., Mullen, P., Ogloff, J., Thomas, S., Wells, D., \& Spataroc, J. (2010). Psychopathology in a large cohort of sexually abused children followed up to 43 years. Child Abuse \& Neglect, 34, 813-822.

http://dx.doi.org/10.1016/j.chiabu.2010.04.004

Draucker, C. B., Martsolf, D., Roller, C., Knapik, G., Ross, R., \& Stidham, A. (2011). Healing from childhood sexual abuse: A theoretical model. Journal of Child Sexual Abuse, 20(4), 435-466. http://dx.doi.org/10.1080/10538712.2011.588188

Echeburúa, E. y Guerricaechevarría, C. (2005). Concepto, factores de riesgo y efectos psicopatológicos del abuso sexual infantil. En J. San Martín (Ed.), Violencia contra los niños (pp. 86-112). Barcelona: Ariel.

Echeburúa, E. y Subijana, J. (2008). Guía de buena práctica psicológica en el tratamiento judicial de los niños abusados sexualmente. International Journal of Clinical and Health Psychology, 8(3), 733-749.

Estrada, L. (2012). Protección de los derechos humanos de las víctimas de abuso sexual: una mirada desde la jurisprudencia. Trabajo Social, 14, 179-191. 
Fondo de las Naciones Unidas para la infancia, Unicef. (2012). Cuarto estudio de maltrato infantil. Santiago: United Nations Children's Fund, Unicef.

Foster, J. \& Hagedorn, B. (2014). Through the eyes of the wounded: A narrative analysis of children's sexual abuse experiences and recovery process. Journal of Child Sexual Abuse, 23(5), 538-557.

http://dx.doi.org/10.1080/10538712.2014.918072

Guerra, C., Viveros, M., Calvo, B., Canessa, P. y Mascayano, F. (2011). Niveles de ansiedad en niños victimizados sexualmente que deben declarar en juicios orales: aportes de un programa de preparación. Revista de Psicología, 20(2), 7-24.

http://dx.doi.org/10.5354/0719-0581.2011.17930

Lamb, M. E., Orbach, Y., Hershkowitz, I., Esplin, P. W., \& Horowitz, D. (2007). A structured forensic interview protocol improves the quality and informativeness of investigative interviews with children: A review of research using the NICHD Investigative Interview Protocol. Child Abuse and Neglect, 31(11-12), 1201-1231.

http://dx.doi.org/10.1016/j.chiabu.2007.03.021

Lipovsky, J. \& Stern, P. (1997). Preparing children for court: An interdisciplinary view. Child Maltreat, 2(2), 150-163.

http://dx.doi.org/10.1177/1077559597002002008

Maffioletti, F. y Huerta, S. (2011). Aproximación fenomenológica de los delitos sexuales en Chile: la realidad nacional. Revista Jurídica del Ministerio Público de Chile, 47, 1-15.

Martínez, J. (2012). Abuso sexual infantil ¿qué se repara en la terapia? En M. C. Aretio y M. Heresi (Eds.), Temas de psicología clínica infanto-juvenil: aportes y reflexiones (pp. 95-111). Santiago de Chile: Universidad Diego Portales.

Michels, A. (2009). Psychosocial support for children: Protecting the rights of child victims and witnesses in transitional justice processes (Innocenti working paper $\mathrm{N}^{\mathrm{o}} 2010$ 14). Florence: Unicef Innocenti Research Centre.

Ministerio Público de Chile. (2014). Boletín estadístico III trimestre (documento de trabajo interinstitucional). Recuperado de http://www.fiscaliadechile.cl/Fiscalia/estadisticas/index.do

Miranda, M. (2012). Victimización secundaria en adolescentes víctimas de delitos sexuales en su paso por el sistema procesal penal en Chile: una aproximación narrativa (Tesis para optar al grado de Magíster en Psicología, mención Psicología Clínica InfantoJuvenil). Universidad de Chile, Chile.

Núcleo de Investigación Infancia y Justicia (2015). Proyecto Fondef. Recuperado de http://infanciayjusticia.uchile.cl/proyecto-fondef/

Pereda, N., Guilera, G., Forns, M., \& Gómez-Benito, J. (2009). The international epidemiology of child sexual abuse: A continuation of Finkelhor (1994). Child Abuse \& Neglect, 33, 331-342.

http://dx.doi.org/10.1016/j.chiabu.2008.07.007

Plotnikoff, J. \& Woolfson, R. (2001). An evaluation of child witness support. Edinburgh: The Scottish Executive Central Research. 
Powell, M. B. (2008). Designing effective training programs for investigative interviewers of children. Current Issues in Criminal Justice, 20(2), 189-208.

Putnam, F. (2003). Ten-year research update review: Child sexual abuse. Journal of the American Academy of Child and Adolescent Psychiatry, 42(3), 269-278. http://dx.doi.org/10.1097/00004583-200303000-00006

Riessman, C. K. (2008). Narrative methods for the human sciences. California: Sage Publications.

Servicio Nacional de Menores, Sename. (2012). Bases técnicas específicas: programa de protección especializado modalidad maltrato infantil grave y abuso sexual. Recuperado de http://www.sename.cl/wsename/licitaciones/p7_27-02-

2012/PRM\%20Bases\%20Tecnicas\%20Licitac\%20\%20Febrero\%202012.pdf

Valles, M. (2003). Técnicas cualitativas de investigación social. Madrid: Ed. Síntesis. 